\title{
Issues and Events
}

\section{ADA Events}

Ohio CME Program

1 November 2012

Embassy Suites

Independence, Ohio

Contact: Joann Mraz

E-mail: jmraz@diabetes.org

Web site: http://professional.diabetes.org/ohio

43rd Annual Symposium for Healthcare Providers

7 November 2012

Crowne Plaza Hotel

Cromwell, Connecticut

Contact: Louise Butcher

E-mail: lbutcher@diabetes.org

Web site: http://professional.diabetes.org/ashcp

1st American Diabetes Association Middle East Congress

4-6 December 2012

Dubai International Convention and Exhibition Centre

Dubai, United Arab Emirates

Web site: http://ada-me.org/index.html

Contact for information: EDC, Center for Diabetes Education,

3119 Trees of Avalon Parkway, McDonough, GA 30253.

Tel: 678-759-1561. E-mail: info@ada-me.org 60th Annual Advanced Postgraduate Course

22-24 February 2013

Hilton New York

New York, New York

Web site: http://professional.diabetes.org/pg13

73rd Scientific Sessions

21-25 June 2013

McCormick Place Convention Center

Chicago, Illinois

Web site: http://scientificsessions.diabetes.org

74th Scientific Sessions

13-17 June 2014

Moscone Center

San Francisco, California

Web site: http://scientificsessions.diabetes.org

Contact for information on ADA events: American Diabetes Association, 1701 N. Beauregard St., Alexandria, VA 22311. Tel: 800-232-3472, select option 1. Fax: 703-549-1715 or 703-253-4358. E-mail: professionaleducation@diabetes.org. Web site: http://professional.diabetes.org/ce.

\section{Other Events}

4th World Congress on Controversies to Consensus in Diabetes, Obesity and Hypertension (CODHy)

8-11 November 2012

Barcelona, Spain

Web site: http://www.codhy.com/2012/

The 2nd International Conference on Prehypertension and Cardio Metabolic Syndrome (PreHT 2013)

31 January-3 February 2013

Barcelona, Spain

Web site: www.prehypertension.org 